\title{
Healable, memorizable, and transformable lattice structures made of stiff polymers
}

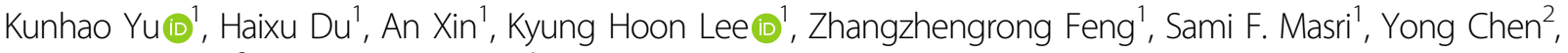 \\ Guoliang Huang ${ }^{3}$ and Qiming Wang ${ }^{1}$
}

\begin{abstract}
Emerging transformable lattice structures provide promising paradigms to reversibly switch lattice configurations, thereby enabling their properties to be tuned on demand. The existing transformation mechanisms are limited to nonfracture deformation, such as origami, instability, shape memory, and liquid crystallinity. In this study, we present a class of transformable lattice structures enabled by fracture and shape-memory-assisted healing. The lattice structures are additively manufactured with a molecularly designed photopolymer capable of both fracture healing and shape memory. We show that 3D-architected lattice structures with various volume fractions can heal fractures and fully restore stiffness and strength over two to ten healing cycles. In addition, coupled with the shape-memory effect, the lattice structures can recover fracture-associated distortion and then heal fracture interfaces, thereby enabling healing of lattice wing damages, mode-l fractures, dent-induced crashes, and foreign-object impacts. Moreover, by harnessing the coupling of fracture and shape-memory-assisted healing, we demonstrate reversible configuration transformations of lattice structures to enable switching among property states of different stiffnesses, vibration transmittances, and acoustic absorptions. These healable, memorizable, and transformable lattice structures may find broad applications in next-generation aircraft panels, automobile frames, body armor, impact mitigators, vibration dampers, and acoustic modulators.
\end{abstract}

\section{Introduction}

Precisely, architected lattice structures with extraordinary properties, including low density, high specific stiffness, high specific strength, and high energy absorption, have been used in a broad range of engineering applications such as aerospace panels, impact absorbers, acoustic modulators, thermal exchangers, battery electrodes, and biomedical scaffolds ${ }^{1-9}$. A key limitation in most existing lattice structures is that their properties and functions may not be modulated once fabricated. A promising direction in the field is designing transformable lattice structures whose configurations can be reversibly switched to enable tunable properties ${ }^{10,11}$. Existing

\footnotetext{
Correspondence: Qiming Wang (qimingw@usc.edu)

${ }^{1}$ Sonny Astani Department of Civil and Environmental Engineering, University of Southern California, Los Angeles, CA 90089, USA

2Epstein Department of Industrial and Systems Engineering, University of

Southern California, Los Angeles, CA 90089, USA

Full list of author information is available at the end of the article
}

transformation mechanisms primarily rely on nonfracture deformation such as origami ${ }^{12-14}$, instability ${ }^{15-17}$, shape memory $^{18-20}$, and liquid crystallinity ${ }^{21}$. Fractures have rarely been harnessed to transform lattice structures, because fractures have long been considered a failure mode that compromises the structural integrity and properties; furthermore, healing fractures is also typically challenging for three-dimensional (3D)-architected lattice structures. However, fractures and the corresponding healing, if successfully realized to transform lattice structures, would greatly benefit a broad range of engineering applications in two aspects. First, damage to lattice structures may be intelligently managed and recovered: examples include lightweight panel structures that may recover from foreign-object-impact-induced damage, body armor that may self-repair damage induced by bullets or other shrapnel on the battlefield, lattice dampers that may regain damping properties after healing overload crashes, and biomedical scaffolds that 
may imitate self-healable bones. Second, fracture healing can be a new tool to intelligently tune the lattice connectivity, thereby reversibly switching the static or dynamic properties of lattice structures.

Despite the great potential, the realization of fracture healing in lattice structures is still challenging, primarily due to two technical barriers. First, innovating materials feasible for manufacturing self-healable lattice structures is challenging. Taking photopolymerization-based additive manufacturing (e.g., stereolithography $(\mathrm{STL})^{22,23}$, polyjet $^{19,24}$, self-propagation photopolymer waveguides ${ }^{25,26}$, and two-photon lithography ${ }^{27,28}$ ) as an example, the required material should be both photocurable and self-healable; this kind of material remains largely unexplored. Although $\mathrm{Yu}$ et al. ${ }^{29}$ recently invented photocurable and self-healable elastomers, the stiffnesses of these materials are relatively low $(10-50 \mathrm{kPa})$, making them unsuitable for the application of force-sustaining lattice structures. In addition, although high-strength self-healable polyurethane has been reported recently ${ }^{30,31}$, how to molecularly tailor high-strength self-healable polyurethane to enable photocuring for additive manufacturing remains largely elusive. Second, healing of lattice structures requires precise contact or alignment of fracture interfaces, whereas damage to lattice structures is typically associated with shape change around fracture locations. The fractures cannot be healed properly without the contact of fracture interfaces. Existing healing experiments of self-healing bulk materials typically rely on manual contact of fracture interfaces $^{32-35}$; however, manual contact is challenging for deep cracks or complex lattice architectures. Consequently, the development of transformable lattice structures that can heal fractures is still an outstanding engineering challenge.

In this study, we present a class of transformable lattice structures enabled by fracture and shape-memoryassisted healing. The lattice structures are additively manufactured via a projection STL system (Supplementary Fig. S1) with a polymer ink that features acrylate groups for photocuring and disulfide groups for fracture healing. The printed solid features a Young's modulus as high as $500 \mathrm{MPa}$, similar to that of a typical Teflon $(200-600 \mathrm{MPa})^{36}$. We show that 3D-architected lattice structures with various volume fractions can heal fractures and fully restore stiffness and strength over two to ten healing cycles. In addition, coupled with the shapememory effect, damaged lattice structures can first recover fracture-associated shape changes to align and then heal the fracture interfaces, thereby enabling healing of lattice wing damages, mode-I fractures, dent-induced crashes, and foreign-object-impact-induced damages. Moreover, by harnessing the coupling of fracture and shape-memory-assisted healing, we demonstrate reversible configuration transformations of lattice structures to enable switching among property states of different stiffnesses, vibration transmittances, and acoustic absorptions. Equipped with coupled features, including additive manufacturing, fracture healing, and shape memory, our lattice structures may open promising avenues for smart lightweight structures that can reversibly transform architectures and recover damage through fracturememory-healing cycles. These healable, memorizable, and transformable lattice structures may find broad applications in next-generation aircraft panels, automobile frames, body armor, impact mitigators, vibration dampers, and acoustic modulators ${ }^{1-9}$.

\section{Results}

\section{Design principle for the transformable lattice structures}

The design principle for transformable lattice structures enabled by fracture and shape-memory-assisted healing is motivated by limitations in existing lattice structures featuring either shape-memory (Fig. $1 \mathrm{a}-\mathrm{c})^{18-20}$ or selfhealing (Fig. $1 \mathrm{~d}-\mathrm{f})^{29}$. On the one hand, when an external intervention forces a lattice structure to undergo both a fracture and a shape change around the fracture location, a shape memory lattice structure (typically made of a semicrystalline polymer) may recover the shape change in a thermal cycle, whereas the fracture interface cannot be healed properly (Fig. $1 \mathrm{a}-\mathrm{c})^{18-20}$. On the other hand, a fracture-healable lattice structure may heal the fracture interface by reversibly forming dynamic bonds (e.g., disulfide bonds ${ }^{29}$ ), whereas the damage-associated shape change cannot be recovered properly (Fig. 1d-f). Therefore, both types of lattice structures may not fully recover the structural integrity or function.

Herein, we propose a class of lattice structures made of polymers featuring both shape memory and fracture healing (Fig. 1g-i). In a typical working cycle, a damaged lattice structure with both shape change and material fracture undergoes a shape-recovery process to align the fracture interfaces and then a fracture-healing process to fully repair the fracture interfaces (Fig. 1g). In this way, the damaged lattice structures are expected to fully recover the initial structural integrity and function and even enable multiple damage-recovery cycles.

The polymers employed to fabricate the proposed lattice structures are designed based on urethane linkages (-NH-CO-O-) formed from a reaction between isocyanate groups $(-\mathrm{NCO})$ and hydroxyl groups $(-\mathrm{OH})$ (Fig. $1 j(i)$ and Supplementary Figs. S2 and S3) ${ }^{37,38}$. The backbone of the polymer network is constructed by an aromatic diisocyanate (isophorone diisocyanate (IPDI)) and a diol (polytetramethylene ether glycol, PTMEG) via urethane linkages. To enable self-healing properties, we covalently incorporate dynamic disulfide bonds into the network by linking a diol-terminated disulfide ( $\mathrm{HO} \sim \mathrm{S}$ S OH) (Fig. 1j(i) and Supplementary Figs. S2 and S3) ${ }^{39,40}$. 

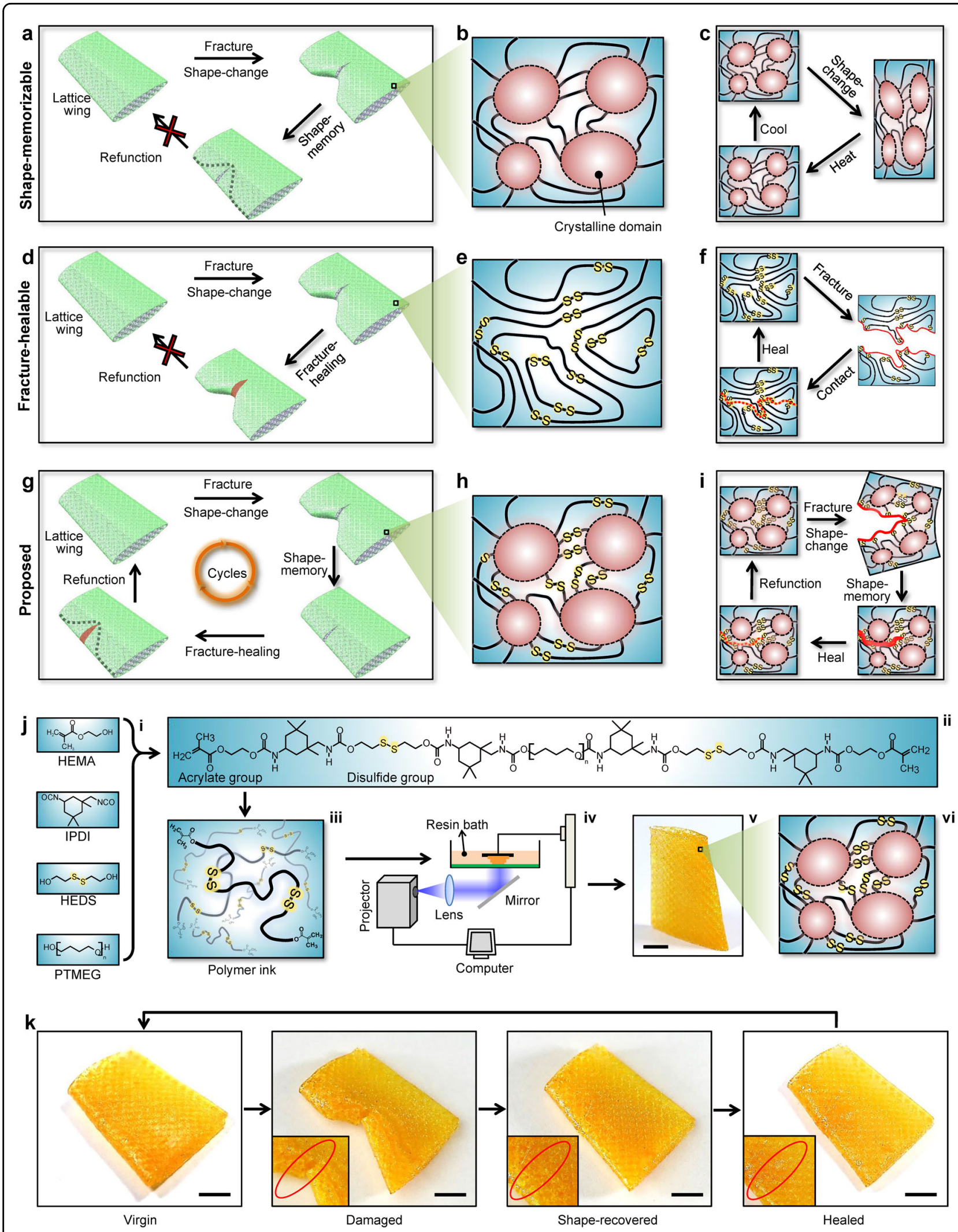

Shape-recovered

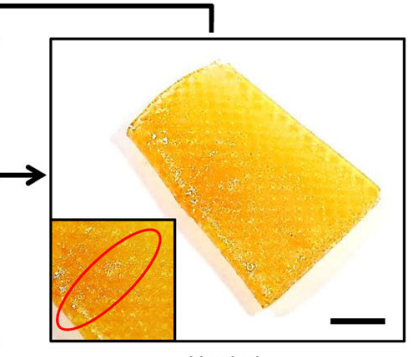

Fig. 1 (See legend on next page.) 
(see figure on previous page)

Fig. 1 Design principle for transformable lattice structures enabled by fracture and shape-memory-assisted healing. a Schematics of the working principle of a shape memory lattice wing structure. b Schematic of the molecular structure of a shape memory semicrystalline polymer. c Schematics of the shape-memory-assisted healing cycle of a shape memory polymer. $\mathbf{d}$ Schematics of the working principle of a fracture-healable lattice wing structure. e Schematic of the molecular structure of a fracture-healable polymer with dynamic disulfide bonds. $\mathbf{f}$ Schematics of the fracture-healing working cycle of a fracture-healable polymer. $\mathbf{g}$ Schematics of the working principle of the proposed lattice wing structure with the coupled properties of shape memory and fracture healing. $\mathbf{h}$ Schematic of the molecular structure of the proposed polymer with both crystalline domains and dynamic disulfide bonds. i Schematics of the working cycle of the proposed polymer. $\mathbf{j}$ Schematics and samples to show the additive manufacturing of a lattice wing: (i) key monomers including 2-hydroxyethyl methacrylate (HEMA) to provide acrylate groups, isophorone diisocyanate (IPDI) to provide isocyanate groups, hydroxyethyl disulfide (HEDS) to provide disulfide groups, and polytetramethylene ether glycol (PTMEG) to provide hydroxyl groups; (ii) chemical formula of the polymer ink with disulfide-linked urethane-acrylate oligomers; (iii) schematic of the polymer ink with disulfide-linked urethane-acrylate oligomers; (iv) schematic of the STL system; (v) lattice wing sample; and (vi) schematic of the molecular structure of the proposed polymer. $\mathbf{k}$ Sample image sequence showing the fracture-memory-healing cycle of a lattice wing. The inset images show the magnified views of the fracture location. The scale bars in $\mathbf{j}(\mathrm{v})$ and $\mathbf{k}$ represent $4 \mathrm{~mm}$.

The self-healing properties primarily rely on disulfide metathesis reactions (assisted by a catalyst tributylphosphine (TBP)) to bridge the fracture interface (Supplementary Fig. S4a $)^{29,41}$. In addition, to enable photocuring properties for STL-based additive manufacturing, we incorporated a hydroxyl-ended acrylate group $\left(\mathrm{CH}_{2}=\right.$ $\mathrm{CHCOO}$ OH) (Fig. 1 j(i) and Supplementary Figs. S2 and $\mathrm{S} 3)^{37}$. The acrylate groups can undergo a photoradicalassisted addition reaction to solidify the polymer (Supplementary Fig. S4b). Thus, the polymer ink for STL is made of disulfide-linked urethane-acrylate oligomers (Fig. 1j(ii-iii)). After photopolymerization, the solid polymer embeds not only dynamic disulfide bonds but also crystalline domains formed through the intermolecular interactions of the polymer chains (Fig. $1 j(v i))^{38}$. The existence of disulfide bonds within the polymer is verified by Raman spectroscopy measurements, which show a new peak with a band at $\sim 520 \mathrm{~cm}^{-1}$ (Supplementary Fig. S5), which was not observed in the control polymer (control 1) without disulfide bonds (Supplementary Fig. S6). This new band is consistent with the Raman band in the reported disulfide-bond-enabled self-healing polymers $\left(500-550 \mathrm{~cm}^{-1}\right)^{29,40,42}$. The existence of the crystalline domain within the polymer is verified by a new endothermic peak at $\sim 130^{\circ} \mathrm{C}$ (Supplementary Fig. S7), which was not observed in the control self-healing polymer (control 2) without crystalline domains ${ }^{29}$. This endothermic peak is consistent with those reported in semicrystalline polyurethanes ${ }^{38}$.

Using a STL system, we can fabricate lattice structures with complex architectures and geometries, and produce the coupled properties of shape memory and fracture healing (Fig. $1 \mathrm{j}(\mathrm{iv}-\mathrm{vi})$ ). The manufacturing process is relatively rapid with a speed of $\sim 25 \mu \mathrm{m} / \mathrm{s}$ for each layer and a total construction time of $\sim 1.5 \mathrm{~h}$ for the lattice wing structure shown in Fig. 1j(v) (Supplementary Fig. S1). As a quick demonstration of a transformable lattice wing in Fig. 1k (Supplementary Movie S1), the lattice wing is first damaged with both a material fracture and a dent. After heating to $80^{\circ} \mathrm{C}$ for $1 \mathrm{~min}$, the dent can be recovered through a shape-memory process, thereby aligning the initially distorted fracture interface. By maintaining the temperature at $80^{\circ} \mathrm{C}$ for another $6 \mathrm{~h}$, the fracture interface can be nicely healed to resume the structural function of the lattice wing. The fracture healing is verified by the magnified pictures and microscope images around the healing interface (inset images in Fig. 1k and Supplementary Fig. S8).

\section{Characterization of shape memory and fracture healing}

Next, we characterize the shape-memory properties of the synthesized polymers. To qualitatively show the shape-memory properties, we first program a twist on a strip sample (with a PTMEG molar mass of $250 \mathrm{~g} / \mathrm{mol}$ ) at $80^{\circ} \mathrm{C}$ and then fix the twist by cooling to room temperature. As the temperature increases again to $80^{\circ} \mathrm{C}$, the twisted sample returns to the initially flat shape within $1 \mathrm{~min}$ (Fig. 2a). The selection of $80^{\circ} \mathrm{C}$ as the recovery temperature is because the glass transition temperature of the polymer is $\sim 65-71^{\circ} \mathrm{C}$ (Supplementary Fig. S9). To quantify the shape-memory properties, we measure the tensile stress-strain behaviors of polymer samples with PTMEG of various molar masses within a thermal cycle (see Materials and Methods). As the molar mass of PTMEG increases, the polymer becomes more flexible with a decreasing glass transition temperature (from above $65-71{ }^{\circ} \mathrm{C}$ for $250 \mathrm{~g} / \mathrm{mol}$ PTMEG to below $25^{\circ} \mathrm{C}$ for $1810 \mathrm{~g} / \mathrm{mol}$ PTMEG; Supplementary Fig. S9). We find that polymers with different glass transition temperatures exhibit different shape-memory cycles (Fig. 2b-d). A typical shape-memory cycle consists of four segments (Fig. 2b-d) as follows: (1) Loading: a polymer sample is uniaxially stretched to a prescribed strain $\varepsilon_{l}$ at $80^{\circ} \mathrm{C}$. (2) Cooling: the strain slightly changes to $\varepsilon_{\mathrm{c}}$ after cooling to $35^{\circ} \mathrm{C}$ under the maintained load. (3) Unloading: the applied load is relaxed at $35^{\circ} \mathrm{C}$ with the strain decreasing to $\varepsilon_{\mathrm{u}}$. (4) Recovering: the temperature increases again to $80^{\circ} \mathrm{C}$ with the strain further decreasing to $\varepsilon_{\mathrm{r}}$. To quantify 
a

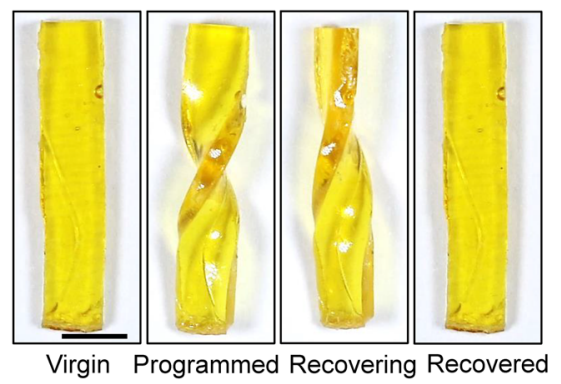

b

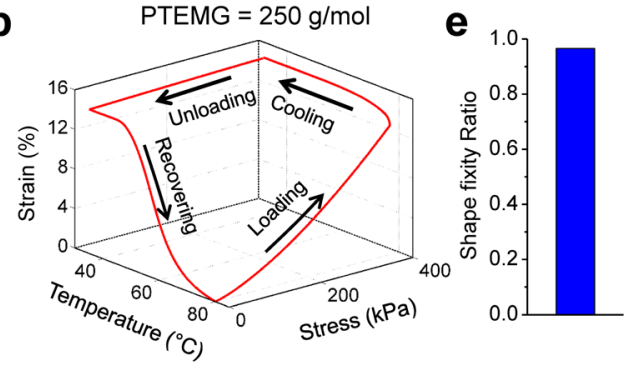

C
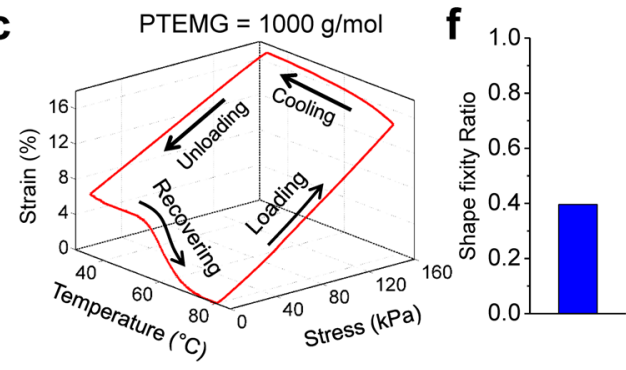

d
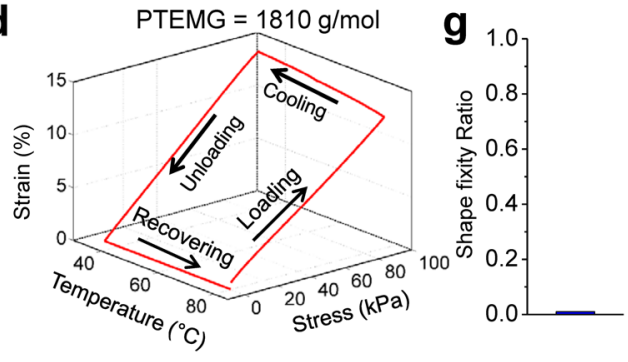

h
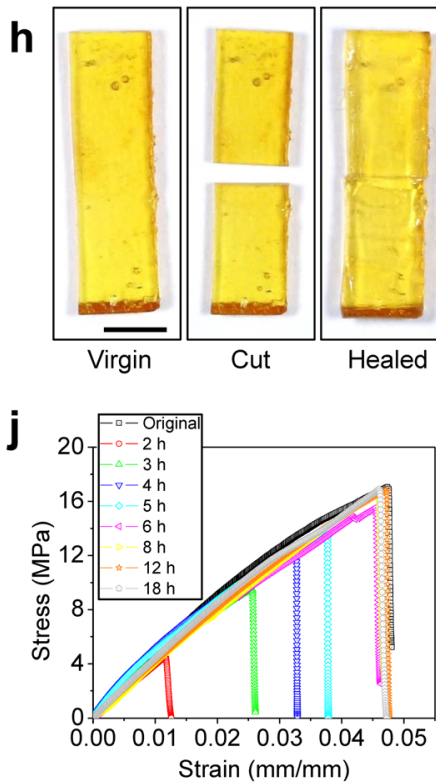

$\mathbf{k}$

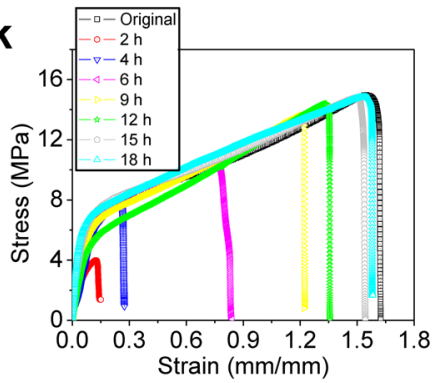

I

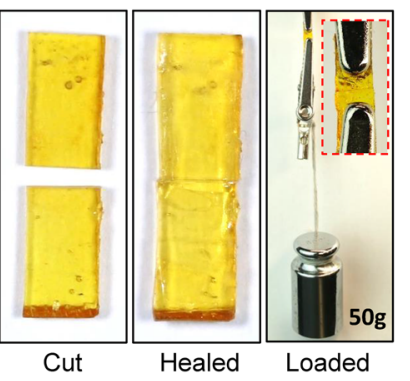

i

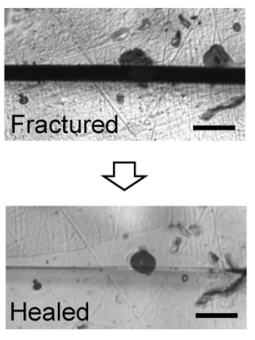

m

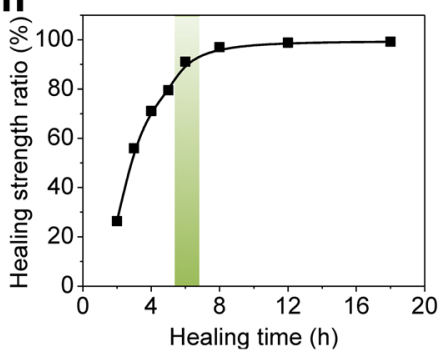

n.

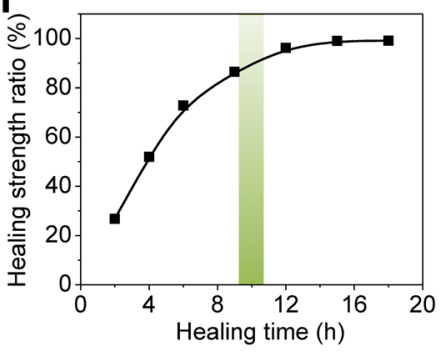

0

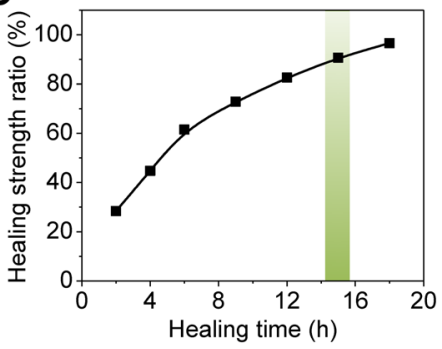

Fig. 2 Characterization of the shape-memory and self-healing properties. a Image sequence showing the shape-memory process of a strip polymer sample. $\mathbf{b}-\mathbf{d}$ Stress-strain-temperature behaviors of synthesized polymers with various PTMEG molar masses within a shape-memory cycle. e- $\mathbf{g}$ Shape-fixity ratios $R_{\mathrm{f}}$ of synthesized polymers corresponding to $\mathbf{b}-\mathbf{d}$. $\mathbf{h}$ Image sequence showing the self-healing process of a strip polymer sample. The healed sample $(0.125 \mathrm{~g})$ can sustain a weight of $50 \mathrm{~g}$. i Microscope images showing fractured and healed interfaces. $\mathbf{j}-\mathbf{I}$ Tensile stress-strain curves of virgin polymers and polymers subjected to various numbers of healing cycles. The polymers in $\mathbf{j}-\mathbf{I}$ have various PTMEG molar masses corresponding to $\mathbf{b}-\mathbf{d}$. $\mathbf{m}$-o Healing strength ratios of healed polymers as a function of healing time. The healing strength ratio is defined as the tensile strength of the healed polymer normalized by the tensile strength of the virgin polymer. The shadow areas in $\mathbf{m}$-o indicate the healing time corresponding to a healing strength ratio of $90 \%$. The scale bars in $\mathbf{a}$ and $\mathbf{h}$ represent $4 \mathrm{~mm}$, whereas the scale bars in $\mathbf{i}$ represent $300 \mu \mathrm{m}$.

the shape-memory properties, we define shape-fixity and shape-recovery ratios as $R_{\mathrm{f}}=\varepsilon_{\mathrm{u}} / \max \left(\varepsilon_{\mathrm{\varepsilon}}, \varepsilon_{\mathrm{c}}\right)$ and $R_{\mathrm{r}}=1-$ $\varepsilon_{\mathrm{r}} / \varepsilon_{\mathrm{u}}$, respectively ${ }^{18-20,38}$. With decreasing glass transition temperature, although the shape-recovery ratio $R_{\mathrm{r}}$ remains at $98-100 \%$, the shape-fixity ratio $R_{\mathrm{f}}$ drastically decreases from $98 \%$ for $250 \mathrm{~g} / \mathrm{mol}$ PTMEG to $1 \%$ for $1810 \mathrm{~g} / \mathrm{mol}$ PTMEG (Fig. 2e-g). Hence, the polymer with the lower PTMEG molar mass exhibits better shapememory properties for a thermal cycle within $35-80^{\circ} \mathrm{C}^{38}$. In addition to the shape-memory properties, the synthesized polymers with disulfide bonds also exhibit selfhealing properties. To qualitatively show this phenomenon, we first cut a strip sample into two parts and then brought these parts into contact at $80^{\circ} \mathrm{C}$ for $6 \mathrm{~h}$ (Fig. 2h). 
The microscopic images show that the fracture interface can be nicely healed (Fig. 2i). The healed strip sample can sustain a weight of $50 \mathrm{~g}$, which is 400 times its own weight $(0.125 \mathrm{~g})$ (Fig. 2h). In contrast, the control polymers (control 1) without disulfide bonds cannot heal the fracture interface after more than $18 \mathrm{~h}$ under the same healing conditions (Supplementary Figs. S6 and S10). To quantify the self-healing properties of disulfide-containing polymers, we carry out uniaxial tensile tests on the virgin polymer strips and healed samples for various healing periods at $80^{\circ} \mathrm{C}$ (Fig. $2 \mathrm{j}-\mathrm{l}$ ). The Young's modulus of the virgin polymer with a PTMEG molar mass of $250 \mathrm{~g} / \mathrm{mol}$ is $\sim 500 \mathrm{MPa}$ (Supplementary Fig. S11), which is within the modulus range of a typical Teflon $(200-600 \mathrm{MPa})^{36}$. As the healing time increases, the tensile strength of the healed sample increases until reaching a plateau, which is the tensile strength of the virgin sample. We take the healing time corresponding to $90 \%$ of the healing strength ratio (tensile strength of the healed sample normalized by that of the original sample) as the equilibrium healing time. We find that the equilibrium healing time increases from $6 \mathrm{~h}$ to $15 \mathrm{~h}$ as the PTMEG molar mass increases from 250 to $1810 \mathrm{~g} / \mathrm{mol}$ (Fig. $2 \mathrm{~m}-\mathrm{o}$ ). This trend can be understood as follows: at $80^{\circ} \mathrm{C}$, which is above the glass transition temperature (Supplementary Fig. S9), the polymer transforms to a rubbery state. The healing process is primarily governed by the coupling of diffusion of polymer chains and the reforming of dynamic disulfide bonds around the healing interface ${ }^{43-45}$. The Rouse diffusivity of a polymer chain is $D=k_{\mathrm{B}} T /(n \xi)$, where $n$ is the Kuhn segment number (understood as the chain length) of the amorphous polymer chain with the disulfide bond, $\xi$ is the Rouse friction coefficient, $k_{\mathrm{B}}$ is the Boltzmann constant, and $T$ is the temperature ${ }^{46,47}$. As the chain length increases (corresponding to increasing PTMEG molar mass), the diffusivity decreases and the required healing time is expected to increase.

Based on the characterization of the shape-memory and self-healing properties, we conclude that to obtain desirable shape-memory and efficient self-healing properties, we should design a polymer with a small PTMEG molar mass. Herein, we selected a polymer with a PTMEG molar mass of $250 \mathrm{~g} / \mathrm{mol}$, which features excellent shapememory properties for a thermal cycle within $35-80^{\circ} \mathrm{C}$ and more than $90 \%$ healing within $6 \mathrm{~h}$ at $80^{\circ} \mathrm{C}$.

\section{Manual-contact-assisted healing of lattice structures}

Next, we study the healing behavior of the lattice structures (Fig. 3). We first fabricate $1 \times 1 \times 4$ octet trusses with relative densities $\rho / \rho_{0}$ from $13.1 \%$ to $53 \%$ ( $\rho$ is the effective lattice density and $\rho_{0}$ is the material density; Fig. 3a(i)) and use a three-point-bending (3PB) load to fracture the lattices (Fig. 3a(ii-iii) and Supplementary Fig. S12). The effective Young's modulus (3.2-10.8 MPa) and flexural strength $(0.7-3.1 \mathrm{MPa})$ of the octet trusses with various relative densities can be obtained from the 3PB tests (Fig. 3b-e and Supplementary Figs. S12 and S13). We find that the effective Young's modulus $E$ and flexural strength $S$ of the octet trusses are approximately linear functions of their relative densities (Fig. 3f, g), which can be expressed as

$$
\begin{gathered}
\frac{E}{E_{0}} \approx 0.055\left(\frac{\rho}{\rho_{0}}\right) \\
\frac{S}{S_{0}} \approx 0.318\left(\frac{\rho}{\rho_{0}}\right)
\end{gathered}
$$

where $E_{0}$ and $S_{0}$ are the Young's modulus and flexural strength (tensile strength) of the parent polymer with a PTMEG molar weight of $250 \mathrm{~g} / \mathrm{mol}$, respectively. $E_{0}$ and $S_{0}$ can be obtained from Fig. 2 j. These linear relationships (Eqs. (1) and (2)) are consistent with the reported theoretical prediction for the stretching-dominant octet truss $^{48}$.

Two fractured parts of the lattice structures are then brought into contact and placed in a glass container to maintain the contact (Supplementary Fig. S14). After $6 \mathrm{~h}$ at $80^{\circ} \mathrm{C}$, the fracture interfaces are self-repaired through disulfide-enabled interfacial healing (Fig. 3a(iv) and Supplementary Fig. S4a), which is verified by microscopic images of the fracture interface (Fig. 3a(v-vi)). The healed lattice can sustain a weight of $70 \mathrm{~g}$, which is $\sim 400$ times its own weight $(0.174 \mathrm{~g})$ (Fig. 3a(vii)). Then, the healed lattice can be fractured by the 3 PB load again and the resulting fracture location is different from that of the first fracture (Fig. 3a(viii)). We find that the effective Young's moduli and flexural strength of the healed octet lattices (firsthealed lattices) can reach above $90 \%$ of those of the virgin lattices (Fig. 3b-g and Supplementary Fig. S13). In addition, the fractured first-healed lattices were healed again after $6 \mathrm{~h}$ at $80^{\circ} \mathrm{C}$ (Fig. 3a(ix)). The linear relationships in Eqs. (1) and (2) are still valid for both the first-healed and second-healed lattices (Fig. 3f, g). In this way, the octet lattice with relative densities $\rho / \rho_{0}=13.1 \%$ can be repeatedly fractured and healed over ten cycles (Supplementary Fig. S15). The effective moduli and strength of the healed lattice structures fluctuate within $85 \%-105 \%$ of those of the virgin lattice but do not show evident degradation trends over ten healing cycles. To the best of our knowledge, this is the first demonstration of full healing of the moduli and strength of 3D-architected lattice structures over multiple healing cycles.

\section{Shape-memory-assisted healing of lattice fractures}

Fractures in lattice structures are typically associated with geometrical distortions of fracture surfaces. Without using manual contact, we show that the shape-memory effect of the lattice structure can assist the distorted 
a
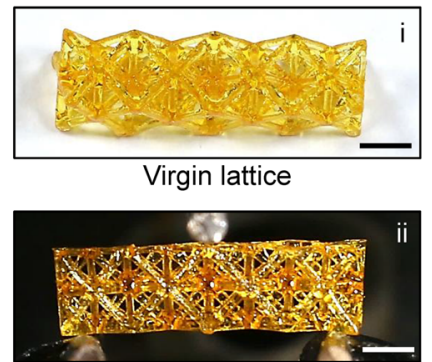

Three-point bending

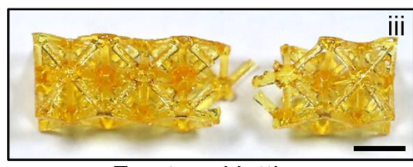

Fractured lattice

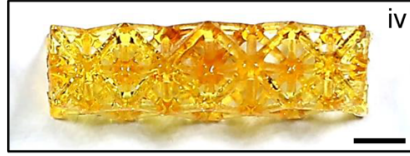

First-healed lattice
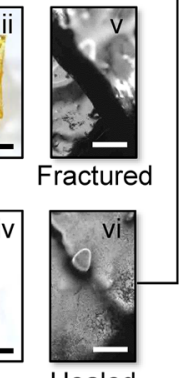

Healed

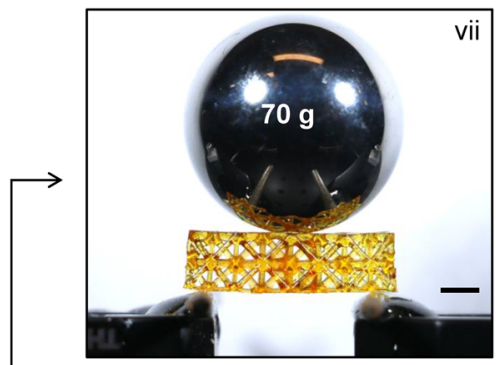

Healed lattice sustaining a weight

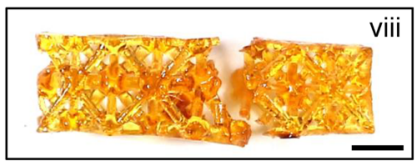

Fracture again

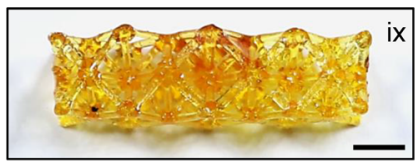

Second-healed lattice
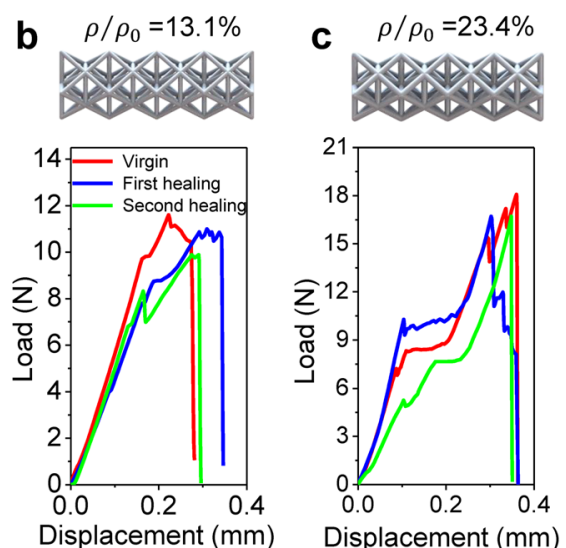

Displacement ( $\mathrm{mm})$
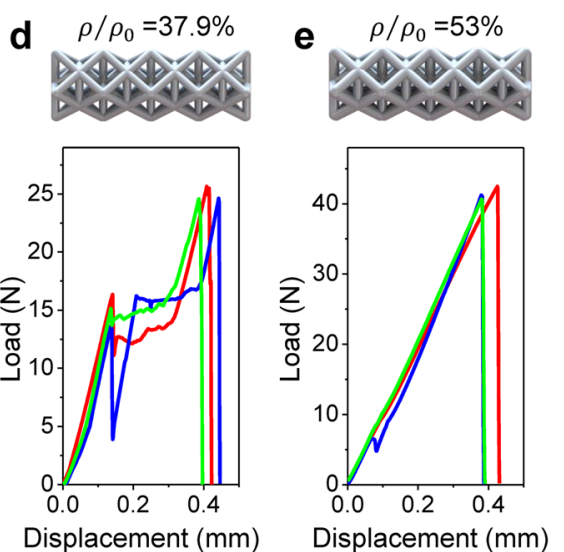
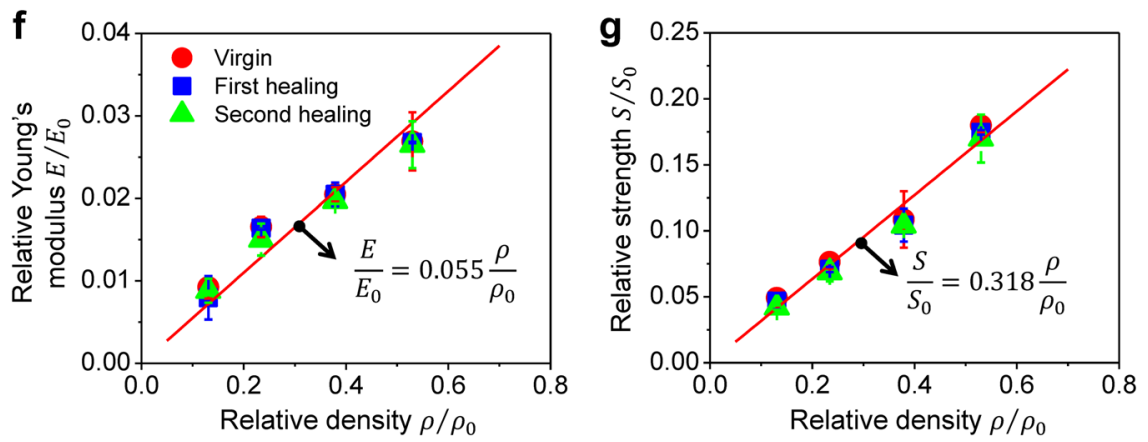

Fig. 3 Manual-contact-assisted healing of lattice structures. a The healing process of an octet lattice over two healing cycles: (i) virgin octet lattice, (ii) octet lattice under a three-point-bending (3PB) load, (iii) fractured octet lattice, (iv) healed octet lattice after the first healing cycle (6 h at $80^{\circ} \mathrm{C}$ ), (v) microscope image showing the fracture interface, (vi) microscope image showing the healed interface, (vii) a mass of $70 \mathrm{~g}$ placed on the healed lattice, (viii) the healed lattice fractured again, and (ix) healed octet lattice after the second healing cycle ( $6 \mathrm{~h}$ at $80^{\circ} \mathrm{C}$ ). The scale bars in $\mathbf{a}$ (i-iv) and $\mathbf{a}$ (vii-ix) represent $4 \mathrm{~mm}$, whereas the scale bars in $\mathbf{a}(\mathrm{v}-\mathrm{vi})$ represent $300 \mu \mathrm{m}$. $\mathbf{b}$-e Computer-aided design models and load-displacement curves of virgin, first-healed, and second-healed octet lattices of various relative densities $\left(\rho / \rho_{0}=13.1 \%, 23.4 \%, 37.9 \%\right.$, and $\left.53 \%\right)$ in $3 P B$ tests. $\mathbf{f}$ The effective Young's moduli of virgin, first-healed, and second-healed octet lattices as functions of the relative density. $\mathbf{g}$ The effective flexural strength values of the virgin, first-healed, and second-healed octet lattices as functions of the relative density. The error bars represent the SDs in three to five samples. 
interface in returning to the initial geometry, through which the fracture interfaces can be aligned and contacted. This process enables the subsequent interfacial fracture healing to be realized. It is noteworthy that the self-alignment of the fracture interface through the shapememory properties can work for complex geometries and deep cracks within the matrix, which are typically challenging for manual contact.

In the first example, a lattice structure is fractured in mode I (Fig. 4a). In the damaged state (Fig. 4a(ii)), the fracture surfaces are separated by a frozen crack width $d$. If the fracture surfaces do not contact each other, the fracture cannot be healed. We first increase the temperature to $80^{\circ} \mathrm{C}$ to trigger a shape-memory process, which enables the crack width to gradually decrease to zero within $1 \mathrm{~min}$ (Fig. 4a(ii-iv), 4b, Supplementary Fig. S16, and Supplementary Movie S2). It is noteworthy that the fracture interface has not been healed in this stage. Then, we maintained the temperature $\left(80^{\circ} \mathrm{C}\right)$ for $6 \mathrm{~h}$ until the fracture interface was fully healed (Fig. $4 \mathrm{a}(\mathrm{v}))$. The healing is verified by microscope images of the fracture interface of a beam before and after the healing process (Fig. 4a(vi-vii)). To further verify the fracture healing, we apply a 3 PB load to the healed interface (Fig. 4c). We find that the maximal 3PB load of the healed lattice (Fig. 4a(v)) is $>90 \%$ of that of the virgin lattice (Fig. $4 \mathrm{c}$ ), whereas the maximal 3PB load of the shape-recovered lattice with an unhealed fracture (Fig. $4 \mathrm{a}(\mathrm{iv})$ ) is only $20 \%$ of that of the virgin lattice (Fig. 4c).

In the second example, a circular indenter is loaded onto a lattice structure to induce a geometrical dent with a certain depth $h$ (Fig. $4 \mathrm{~d}(\mathrm{i}-\mathrm{iii})$ ). The magnified picture shows microfractures within the internal beams (Fig. $4 \mathrm{~d}$ (iii)). Harnessing the shape-memory effect at $80^{\circ} \mathrm{C}$, the dent depth can be recovered in $1.5 \mathrm{~min}$, thereby enabling the alignment of the fracture surfaces of the internal beams (Fig. 4d(iv), e, Supplementary Fig. S17 and Supplementary Movie S3). Subsequently, additional healing for $6 \mathrm{~h}$ at $80^{\circ} \mathrm{C}$ can further repair the interfacial microcracks in the beams. The healing is verified by microscope images of the fracture interface of a beam before and after the healing process (Fig. $4 \mathrm{~d}$ (vi-vii)). To further verify the fracture healing, we use the indenter to test the structural stiffness and find that the stiffness of the healed lattice (Fig. $4 \mathrm{~d}(\mathrm{v})$ ) is approximately equal to that of the virgin lattice, whereas the stiffness of the lattice with the recovered shape but unhealed fractures (Fig. $4 \mathrm{~d}(\mathrm{iv})$ ) is only $\sim 17 \%$ of that of the virgin lattice (Fig. $4 \mathrm{f}$ ).

In the third example, we demonstrate that the transformable lattice can be programmed to intelligently recover impact-induced damage (Fig. 4g, h). If the impact force is relatively small and only induces a dent in the lattice structure, the dent can be removed through a shape-memory process (Supplementary Fig. S18 and
Supplementary Movie S4). If the impact force is relatively large and induces a punch-through hole with spike fractures (Fig. 4g, $\mathrm{h}(\mathrm{i}-\mathrm{ii}), \mathrm{i}(\mathrm{i}-\mathrm{ii})$ ), the restoration should rely on the coupling of shape recovery and fracture healing. Aside from some small detached debris, the initial shape is first recovered through a shape-memory process at $80^{\circ} \mathrm{C}$ for $1.5 \mathrm{~min}$ (Fig. $4 \mathrm{~h}(\mathrm{ii}-\mathrm{v}$ ), i(ii-v), Supplementary Fig. S17, and Supplementary Movie S5). Then, the fracture interfaces of the shape-recovered parts are fully healed through a fracture-healing process at $80^{\circ} \mathrm{C}$ for $6 \mathrm{~h}$ (Fig. $4 \mathrm{~h}(\mathrm{v}-\mathrm{vi})$, i (v-vi) and Supplementary Movie S5). Fracture healing is verified by microscopic images before and after the healing process (Fig. 4i (vii-viii)). To demonstrate the advantage of the damage restoration of the lattice structure, we investigate the impact-mitigation behavior of the virgin (Fig. 4i(i)), damaged (Fig. 4i(ii)), and fracture-healed lattices (Fig. 4i (vi)) using the experimental setup shown in Fig. 4j. We employ a weight $(50 \mathrm{~g})$ dropped from a height of $5 \mathrm{~cm}$ to impact the lattice structures and measure the reaction force beneath the lattice structures (Fig. 4k). We find that the reaction force beneath the virgin lattice is only $16.2 \%$ (reaction force ratio) of that without a lattice structure (Fig. 4l). When the lattice is damaged, the reaction force ratio drastically increases to $94 \%$ (Fig. 4l). However, when the lattice is healed, the reaction force ratio decreases again to $16.6 \%$ (Fig. 4l).

\section{Lattice transformation via fracture-memory-healing cycles}

Next, we show that harnessing fracture-memoryhealing cycles can enable on-demand transformation of lattice configurations and subsequently lead to intelligent switching of static or dynamic mechanical properties of lattice structures (Fig. 5). Take a honeycomb lattice as the first example: the stiffness primarily comes from the contribution of the force-sustaining vertical beams (Fig. 5a(i), b(i)). After deactivating five vertical beams via fracture and bending (Fig. 5a(ii), b(ii)), the lattice exhibits a stiffness of $0.96 \mathrm{MPa}$, which is $22 \%$ lower than that of the virgin lattice (1.2 MPa) (Fig. $5 \mathrm{c}, \mathrm{d})$. Then, the deactivated beams can be fully healed through a memoryhealing process (Fig. 5a(iii), b(iii)), thereby recovering $100 \%$ of the stiffness of the virgin lattice (Fig. 5d). The lattice can be further transformed to a state with ten beams deactivated by cutting and bending, which is associated with a 59\% stiffness reduction (Fig. 5a(iv), b(iv), e). Then, the lattice can be restored to return the stiffness to $1.1 \mathrm{MPa}$, which is $92 \%$ of the stiffness of the virgin lattice (Fig. 5e).

In the second example, we show that the fracturememory-healing cycle can enable lattice transformation and subsequent reversible switching of vibration transmittance of lattice structures. A triangle lattice can be transformed to a Kagome lattice if the horizontal beams 


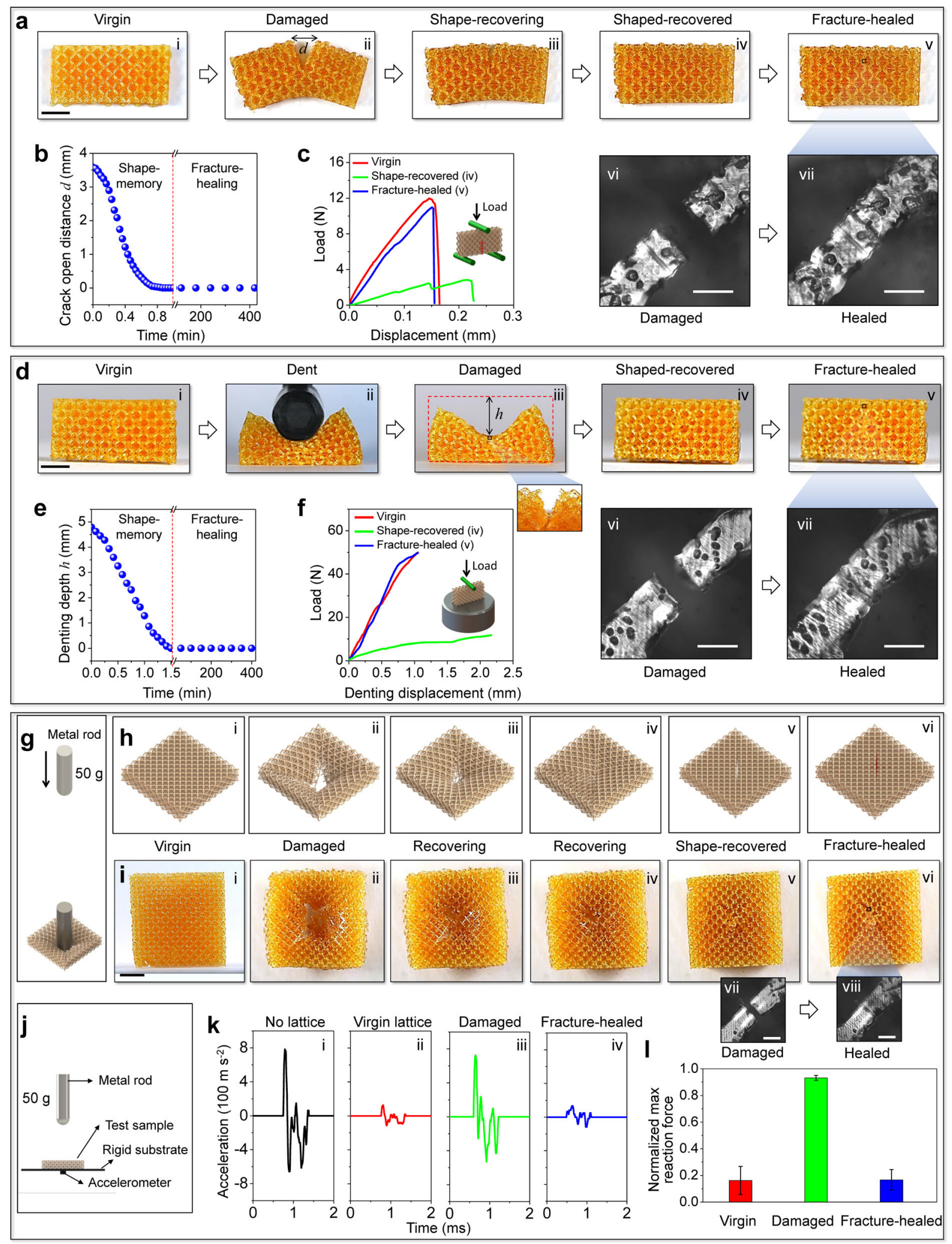

Fig. 4 (See legend on next page.) 
(see figure on previous page)

Fig. 4 Shape-memory-assisted healing of lattice fractures. a Shape-memory-assisted healing of a mode-l fracture in an octet lattice: (i) virgin lattice, (ii) damaged lattice with a frozen mode-l fracture, (iii) shape-recovering lattice, (iv) shape-recovered lattice with fracture interfaces, (v) healed lattice with recovered shape and healed fracture, (vi) microscope image of a fractured lattice beam, and (vii) microscope image of a healed lattice beam. $\mathbf{b}$ Crack width as a function of time during the shape-memory and fracture-healing processes. $\mathbf{c}$ Load-displacement curves of the virgin, shaperecovered with fracture interfaces, and fracture-healed lattices in 3PB tests; the inset shows the 3PB test setup. $\mathbf{d}$ Shape-memory-assisted healing of a dented octet lattice: (i) virgin lattice, (ii) lattice deformed by an indenter, (iii) damaged lattice, (iv) shape-recovered lattice with fractured beams, (v) healed lattice with recovered shape and healed fracture, (vi) microscope image of a fractured lattice beam, and (vii) microscope image of a healed lattice beam. e Dent depth as a function of time during the shape-memory and fracture-healing processes. $\mathbf{f}$ Load-displacement curves of the virgin, shape-recovered with fracture interfaces, and fracture-healed lattices in the denting tests; the inset shows the denting test setup. $\mathbf{g}$ Schematic showing the impact-induced damage of an octet lattice structure. $\mathbf{h}$ Schematic sequence and $\mathbf{i}$ experimental image sequence of shape-memoryassisted healing of the impact-induced damage of an octet lattice: (i) virgin lattice, (ii) impact-induced damaged lattice, (iii-iv) shape-recovering lattice, (v) shape-recovered lattice with fracture interfaces, (vi) fracture-healed lattice, (vii) microscope image of a fractured lattice beam, and (viii) microscope image of a healed lattice beam. $\mathbf{j}$ Experimental setup for testing the reaction forces of impacts on lattice structures, in which the impact is applied by a dropping weight (50 g) from a height of $5 \mathrm{~cm}$. $\mathbf{k}$ The impact reaction forces in the cases with (i) no lattice, (ii) virgin lattice, (iii) damaged lattice, and (iv) fracture-healed lattice. I The normalized maximal reaction forces of the virgin lattice, the damaged lattice, and the fracture-healed lattice. The normalized maximal reaction forces are calculated as the maximal reaction force of each case normalized by the maximal reaction force of the no-lattice case. The error bars represent SDs from five to ten tests. The scale bars in $\mathbf{a}(\mathrm{i}), \mathbf{d}(\mathrm{i})$, and $\mathbf{i}(\mathrm{i})$ represent $4 \mathrm{~mm}$, whereas the scale bars in $\mathbf{a}(v i-v i i), \mathbf{d}(v i-v i i)$, and $\mathbf{i}(v i i-v i i i)$ represent $200 \mu \mathrm{m}$.

are deactivated via fracture and bending (Fig. $5 \mathrm{f}(\mathrm{i}-\mathrm{iii})$ ). According to reported numerical simulations ${ }^{49}$, the triangle lattice displays a band gap in transmitting in-plane elastic waves within a structure-dependent frequency regime, whereas the Kagome lattice does not display any band gap (simulations in Supplementary Fig. S19). Motivated by the numerical simulations (Supplementary Fig. S19), we experimentally measure the in-plane vibration transmittance using the setup shown in Fig. 5g (Supplementary Fig. S20). We find that the triangle lattice exhibits a relatively low vibration transmittance $(<0.2)$ within $32.2-33.2 \mathrm{kHz}$, whereas the transformed Kagome lattice presents a relatively high vibration transmittance $(>0.8)$ within the same frequency regime (Fig. $5 \mathrm{~h}$ ). After heating the programmed Kagome lattice for $\sim 6 \mathrm{~h}$ at $80^{\circ} \mathrm{C}$, the lattice transforms back to the triangle lattice through a coupled process of shape memory and fracture healing (Fig. 5f(iii-iv)). The healing of fracture interfaces can be verified by a magnified picture (Fig. $5 f(v))$. Once back to the triangle lattice, the vibration transmittances within $32.2-33.2 \mathrm{kHz}$ shift to low values $(<0.2)$ again (Fig. $5 \mathrm{~h})$. As an alternative way to present the results, the normalized wave amplitudes of the lattice at three states at $32.75 \mathrm{kHz}$ are shown in Fig. 5i: the programmed Kagome lattice shows a large increase in the wave amplitude and then returns to a small amplitude after restoration to the triangle lattice. It is noteworthy that the restoration of vibration transmittance requires the integration of shape recovery and fracture healing, whereas only shape recovery cannot restore the vibration transmittance property (Supplementary Fig. S21).

In the third example, we show that the structural transformation enabled by the fracture-memory-healing cycle can also switch the acoustic absorption of the lattice structures (Fig. $5 j-m$ ). The key idea here is to reversibly switch local resonators within a lattice structure. The virgin lattice consists of 16 local resonators in which a rectangular island is connected to the structural frame through two thin beams (resonator A; Fig. 5j(i-ii)) $)^{50}$. After deactivating one thin beam via fracture and bending, the island and another thin beam constitute another local resonator (resonator B; Fig. 5j(iii)). The resonances of resonators $\mathrm{A}$ and $\mathrm{B}$ can be triggered by external acoustic waves with different frequencies because of the difference in the resonator structures. The local resonance within the structure can then trap the incoming acoustic wave and significantly lower the acoustic transmittance. To demonstrate the concept, we measure the acoustic transmittances of the virgin and transformed lattice structures using the setup shown in Fig. 5k (Supplementary Fig. S22). We find that the virgin lattice with resonator A (Fig. 5j(ii)) shows a relatively low acoustic transmittance within a frequency of $610-670 \mathrm{~Hz}$; however, the transformed lattice with resonator B (Fig. 5j(iii)) exhibits a dramatic decrease in the acoustic transmittance within a frequency of $280-320 \mathrm{~Hz}$ (Fig. 5l). These two frequency regimes represent the resonance frequencies of resonator $\mathrm{A}$ and resonator $\mathrm{B}$, respectively. The experimental measurements are roughly verified by numerical simulations of the acoustic transmittance of the virgin and transformed lattices (Supplementary Fig. S23). Then, the transformed lattice structure can transform back to the virgin shape via a memory-healing process (Fig. 5j(iv)). The fracture healing is verified by a magnified picture of the fracture interface (Fig. $5 j(v)$ ). After shape recovery and fracture healing, the corresponding acoustic transmittance returns to that of the virgin lattice (Fig. 5l). Overall, at $300 \mathrm{~Hz}$, the transmitted acoustic amplitude can be represented by a high-low-high cycle corresponding to the virgin-programmed-restored cycle of the structure 

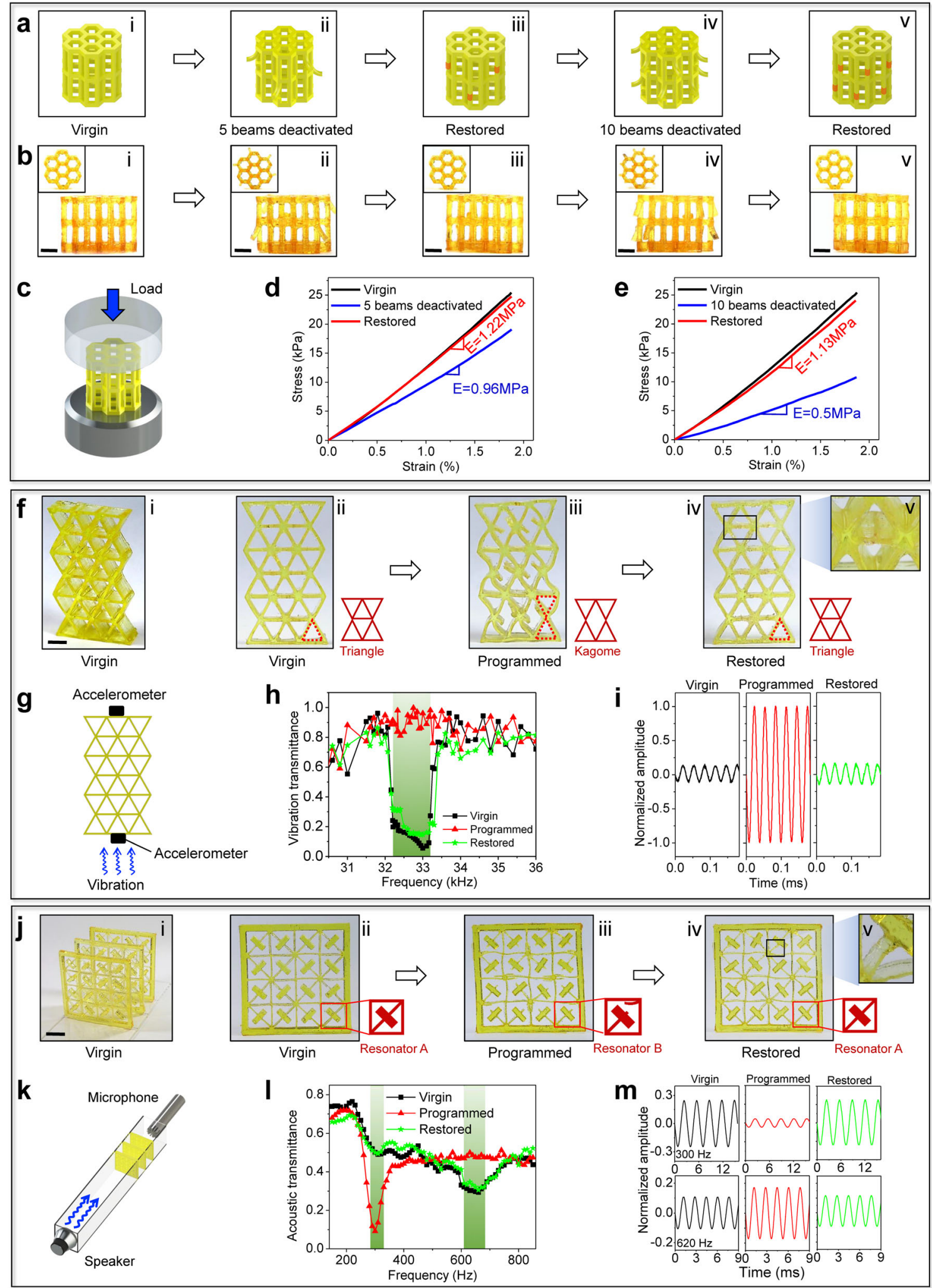

Fig. 5 (See legend on next page.) 
(see figure on previous page)

Fig. 5 Lattice transformation enabled by fracture-memory-healing cycles. $\mathbf{a}$, b Schematics and samples of honeycomb lattices in structural transformation processes: (i) virgin lattice, (ii) lattice with five vertical beams deactivated, (iii) lattice with five deactivated beams restored via shape memory and fracture healing, (iv) lattice with ten vertical beams deactivated, and (v) lattice with ten deactivated beams restored via shape memory and fracture healing. It is noteworthy that the insets in $\mathbf{b}$ show the top view of honeycomb lattices. $\mathbf{c}$ Schematics showing the compression test of a honeycomb lattice. $\mathbf{d}$ Compressive stress-strain curves of the virgin lattice, lattice with five beams deactivated, and lattice with five deactivated beams restored. e Compressive stress-strain curves of the virgin lattice, lattice with ten beams deactivated and ten lattice with deactivated beams restored. $\mathbf{f}$ Transformation between a triangle lattice and a Kagome lattice: (i) 3D view of a virgin triangle lattice, (ii) $2 \mathrm{D}$ view of the virgin triangle lattice, (iii) programmed Kagome lattice by cutting and bending the horizontal beams of the triangle lattice, (iv) restored triangle lattice, and (v) a magnified image to show the healed interface. Note that the insets show representative cells of the triangle and Kagome lattices. $\mathbf{g}$ Experimental setup for testing the vibration transmittances of lattice structures. $\mathbf{h}$ Measured vibration transmittances of the virgin triangle lattice, programmed Kagome lattice and restored triangle lattice as functions of the vibration frequency. i Normalized vibration amplitudes of the virgin triangle lattice, programmed Kagome lattice and restored triangle lattice at $32.75 \mathrm{kHz}$. $\mathbf{j}$ Transformation between a lattice with resonator $A$ and a lattice with resonator B: (i) 3D view of an array of virgin lattices with resonator A, (ii) 2D view of the virgin lattice with resonator A, (iii) programmed lattice with resonator B, (iv) restored lattice with resonator $A$, and (v) a magnified image to show the healed interface. Note that the insets show unit cells of resonators $A$ and B. $\mathbf{k}$ Experimental setup for testing the acoustic transmittances of lattice structures. I Measured acoustic transmittances of the virgin, programmed, and restored lattices as functions of the acoustic frequency. $\mathbf{m}$ Normalized acoustic amplitudes of the virgin, programmed, and restored lattices at $300 \mathrm{~Hz}$ and $620 \mathrm{~Hz}$.

geometry, whereas at $620 \mathrm{~Hz}$, the transmitted acoustic amplitude can be represented by a low-high-low cycle (Fig. 5m).

\section{Discussion}

It is noteworthy that the fractures in Fig. 3 and in Figs. 4 and 5 are different. The 3PB-induced fractures in Fig. 3 occur at room temperature; thus, manual contact is required to assist the alignment of the fracture interfaces. However, the fractures and their associated shape change around the fracture locations in Figs. 4 and 5 are programmed at elevated temperatures $\left(80^{\circ} \mathrm{C}\right.$ for Figs. $4 \mathrm{a}-\mathrm{C}$ and 5 , and $60^{\circ} \mathrm{C}$ for Fig. $4 \mathrm{~d}-\mathrm{l}$ ). Elevated temperatures are required to enable the shape-memory process to align the fracture interfaces via externally controlled thermal stimuli rather than manual contact. This elevatedtemperature requirement is widely adopted for shapememory polymers and structures ${ }^{18-20}$. From a practical perspective, the shape-memory-assisted healing of the lattice fracture in Fig. 4 can be realized by judiciously heating the lattice structure when damage or fracture is expected. Fortunately, the polymer with a PTMEG molar mass of $250 \mathrm{~g} / \mathrm{mol}$ does not become too soft at $60-80^{\circ} \mathrm{C}$ but still exhibits a Young's modulus as high as 140-390 MPa (Supplementary Fig. S9a), which is higher than those of most 3D-printable photopolymers (modulus $<100 \mathrm{MPa})^{51}$.

In summary, we present a class of transformable lattice structures enabled by fracture and shape-memoryassisted healing. The presented lattice structures can heal lattice fractures through manual contact or memoryhealing processes. The fracture-memory-healing cycle can further enable reversible transformations of lattice configurations, shifting properties among states of different stiffnesses, vibration transmittances, and acoustic absorptions. We expect that self-healable lattice structures can promote the future exploration of nextgeneration healable and reusable lightweight materials ${ }^{29}$ within blank Ashby material property space ${ }^{1-9}$. In addition, the shape-memory-assisted healing of lattice structures revolutionizes the state-of-the-art healing paradigms that primarily rely on manual contacts to align fracture interfaces. This paradigm may greatly facilitate the healing of undetected cracks or cracks deep within a structure without external tethered intervention, thereby potentially enabling broad applications in nextgeneration aircraft panels, automobile frames, body armor, impact mitigators, vibration dampers, and acoustic modulators ${ }^{1-9}$. Furthermore, the existing transformable structures primarily harness the nonfracture geometrical change of smart materials ${ }^{18-20,52-54}$; the structural transformations enabled by the fracturememory-healing cycles open a unique avenue by adding a fracture-healing tool, probably enabling previously impossible modulation of functionalities.

\section{Materials and methods \\ Materials}

PTMEG (molar masses of 250, 1000, and $1810 \mathrm{~g} / \mathrm{mol}$ ), IPDI, dimethylacetamide (DMAc), dibutyltin dilaurate (DBTDL), 2-hydroxyethyl disulfide (HEDS), 1,4 butanediol, 2-hydroxyethyl methacrylate (HEMA), TBP, phenylbis (2,4,6-trimethylbenzoyl)phosphine oxide (photoinitiator), and Sudan I (photoabsorber) were purchased from SigmaAldrich and were used without further purification.

\section{Preparation of experimental polymer inks}

First, $0.00829 \mathrm{~mol}$ of PTMEG was preheated at $90^{\circ} \mathrm{C}$ and bubbled with nitrogen for $1 \mathrm{~h}$ to remove oxygen and water. After lowering the temperature to $70^{\circ} \mathrm{C}$, the preheated PTMEG was mixed with $7.369 \mathrm{~g}$ IPDI, $5 \mathrm{~g}$ DMac, and $0.15 \mathrm{~g}$ DBTDL by magnetic stirring for $1 \mathrm{~h}$. Then, a 
solution with $2.557 \mathrm{~g}$ HEDS in $20 \mathrm{~g}$ DMac was added dropwise to the mixture by magnetic stirring for another $1 \mathrm{~h}$. After cooling the mixture to $40^{\circ} \mathrm{C}, 2.147 \mathrm{~g}$ HEMA was added and then the mixture was subjected to one more hour of magnetic stirring to complete the reaction. During the whole process, nitrogen was bubbled into the solution. The obtained solution was then placed in a vacuum chamber for $12 \mathrm{~h}$ to evaporate the solvent. To allow additive manufacturing of the polymer ink, the solution was mixed with $1 \mathrm{wt} \%$ photoinitiator, 0.01 $\sim 0.02 \mathrm{wt} \%$ photoabsorber, and $0.1 \mathrm{wt} \% \mathrm{TBP}$, and then stirred for $2 \mathrm{~h}$. To monitor each reaction step during the ink synthesis, we employed a Spectrum Two FTIR Spectrometer (PerkinElmer, USA) to carry out Fouriertransform infrared spectroscopy (FTIR) analyses (Fig. S3). All the samples were scanned in the range of 450 to $4000 \mathrm{~cm}^{-1}$ at a resolution of $0.5 \mathrm{~cm}^{-1}$.

\section{Additive manufacturing}

The projection-based STL system used to manufacture all the samples presented in this study was described elsewhere $^{23,29}$. We first designed 3D structures in computer-aided design software; the models were output as STL files. Each STL file was then sliced into image sequences with a designated spacing in the vertical direction. The images were sequentially projected with $405 \mathrm{~nm}$ wavelength light onto the resin bath, which was filled with a synthesized polymer ink. A motor-controlled printing stage was mounted onto the resin bath with a prescribed liquid height. The light-exposed resin was solidified and bonded onto the printing stage. As the printing stage was lifted, the fresh resin refluxed beneath the printing stage. By lowering the printing stage to a prescribed height and illuminating the resin with another slice image, a second layer was printed and bonded onto the first layer. These processes were repeated to form a $3 \mathrm{D}$-architected structure. It is noteworthy that a Teflon membrane with a low surface tension $(\sim 20 \mathrm{mN} / \mathrm{m})$ was employed to reduce the separation force between the solidified part and the printing window. Fabricated samples were post-cured for $1 \mathrm{~h}$ in a UV chamber, to enable the full photopolymerization of the material, and then the samples were heated for $12 \mathrm{~h}$ at $40^{\circ} \mathrm{C}$ to remove the residual solvent within the material matrix.

\section{Self-healing characterization}

Strip samples (length of $20 \mathrm{~mm}$, width of $5 \mathrm{~mm}$, and thickness of $1 \mathrm{~mm}$ ) were prepared following the method mentioned in the additive manufacturing section. Samples were first cut into two pieces with a sharp blade and then immediately placed in contact, during which the two ends were clamped to ensure good contact during the healing process. The samples were then placed on a hot plate at $80^{\circ} \mathrm{C}$ for various healing times. Both the original and the healed samples were uniaxially stretched until rupture with a strain rate of $0.06 \mathrm{~s}^{-1}$ with a tensile tester (Instron, Model 5942). Microscopic pictures of the healed surfaces were taken using an optical microscope (Nikon ECLIPSE LV100ND). Raman spectroscopy analyses of the experimental and control samples were carried out using a Horiba Raman infrared microscope with a laser excitation wavelength of $785 \mathrm{~nm}$ in the range of 400 to $1500 \mathrm{~cm}^{-1}$.

\section{Shape-memory characterization}

The shape-memory behavior in Fig. 2 was characterized by the thermomechanical cyclic test programmed in a dynamic mechanical analyzer (DMA 850, TA instrument) using the force control mode. The sample was preheated to an equilibration temperature of $80^{\circ} \mathrm{C}$ and then a static force was applied to the sample. The force was continuously held until the temperature cooled to $35^{\circ} \mathrm{C}$. Then, the force was released at a rate of $0.5 \mathrm{~N} / \mathrm{min}$ to an initial preload of $0.001 \mathrm{~N}$. Finally, the sample was heated back to $80^{\circ} \mathrm{C}$ again. To measure the glass transition temperature, the samples were tested with the oscillation temperature ramp program in a dynamic mechanical analyzer (DMA 850, TA instrument) and heated in the range of $20^{\circ} \mathrm{C}$ to $160^{\circ} \mathrm{C}$ at a rate of $5 \mathrm{~min}^{-1}$. The glass transition temperature was determined using the obtained storage modulus curve as introduced in the literature ${ }^{55}$. To verify the existence of crystalline domains, samples with various molar masses of PTMEG $(250,1000$, and $1810 \mathrm{~g} / \mathrm{mol})$ were tested using differential scanning calorimetry (DSC-8000, PerkinElmer). A $5 \mathrm{mg}$ sample was placed in the alumina plate and heated in the range of $30^{\circ} \mathrm{C}$ to $160^{\circ} \mathrm{C}$ at a rate of $10^{\circ} \mathrm{min}^{-1}$ under the flow of ultrahigh purity nitrogen. An empty alumina plate was placed in the other chamber as a reference.

\section{Manual-contact-assisted healing of octet lattices}

The abovementioned additive manufacturing process was used to fabricated $1 \times 1 \times 4$ octet trusses with different densities $\left(\rho / \rho_{0}=13.1 \%, 23.4 \%, 37.9 \%\right.$, and $\left.53 \%\right)$. The printed structures were first subjected to a 3 PB test until fracture (Instron, Model 5942). The damaged samples were brought into contact at $80^{\circ} \mathrm{C}$ and then healed for $6 \mathrm{~h}$ at $80^{\circ} \mathrm{C}$. The healed structures were subjected to another 3PB test until fracture. Then, the sample was healed and broken again until completing ten healing cycles.

\section{Shape-memory-assisted healing of lattice structures}

Octet lattice structures with different shapes were fabricated using the abovementioned additive manufacturing process. The unit cell size is $\sim 2 \mathrm{~mm} \times 2 \mathrm{~mm} \times$ $2 \mathrm{~mm}$. The lattices in Fig. $4 \mathrm{a}-\mathrm{d}$ feature $2 \times 4 \times 8$ unit cells. 
The lattices in Fig. 4h, i feature $2 \times 9 \times 9$ unit cells. For the mode-I fracture example in Fig. 4a, we employed an electric cutter (WORX WX081L) to cut the lattice structure in half at the center of the sample width, bent the sample to open the crack to $\sim 3.6 \mathrm{~mm}$ at $80^{\circ} \mathrm{C}$, and froze the bending deformation by cooling to room temperature. The damaged lattice, with both shape change and material fracture, was heated to $80^{\circ} \mathrm{C}$ on a hot plate to allow the crack width to close within $1 \mathrm{~min}$. The corresponding shape-memory temperature cycle is shown in Fig. 2b. The temperature of $80^{\circ} \mathrm{C}$ was maintained for an additional $6 \mathrm{~h}$ to enable fracture healing. The loaddisplacement curves of the original (Fig. 4a(i)), shapedrecovered but fractured (Fig. 4a(iv)), and fracture-healed lattices (Fig. $4 \mathrm{a}(\mathrm{v})$ ) in the 3PB tests were obtained by the Instron mechanical tester. For the denting example in Fig. $4 \mathrm{~d}$, a steel rod (diameter $8 \mathrm{~mm}$ ) was employed to dent the lattice at $60^{\circ} \mathrm{C}$ until the internal beams were fractured. The experimental procedures for the shape recovery and fracture healing at $80^{\circ} \mathrm{C}$ were the same as those of the mode-I fracture example in Fig. 4a. The corresponding shape-memory temperature cycle is shown in Supplementary Fig. S17. Denting tests were used to measure the load-displacement curves of the original (Fig. 4d(i)), shape-recovered but fractured (Fig. $4 \mathrm{~d}(\mathrm{iv})$ ), and fracture-healed lattices (Fig. $4 \mathrm{~d}(\mathrm{v}))$ in the central region. In Fig. $4 \mathrm{i}$, a rod with a spherical end $(50 \mathrm{~g})$ was dropped from a height of $1 \mathrm{~m}$ onto the sample at $60^{\circ} \mathrm{C}$. The experimental procedures for the shape recovery and fracture healing at $80^{\circ} \mathrm{C}$ were the same as those of the mode-I fracture example in Fig. 4a. The corresponding shape-memory temperature cycle is shown in Supplementary Fig. S17. An impactmitigation test was performed by dropping a $50 \mathrm{~g}$ rod from a height of $5 \mathrm{~cm}$ onto a rigid plastic substrate. An accelerometer (352C22, PCB Piezotronics, USA) was attached beneath the substrate to measure the reaction acceleration during the impact. The signal was collected with an oscilloscope (TBS 1052B-EDU, Tektronix) when no, virgin, damaged, and healed lattices were placed on the substrate.

\section{Stiffness transformation of honeycomb lattices}

Honeycomb lattice structures were first fabricated using the abovementioned additive manufacturing process. A sharp blade was used to cut the selected vertical beams, which were then deactivated by bending at $80^{\circ} \mathrm{C}$; then, the shape was frozen by cooling to room temperature. The programmed lattice structures were heated to $80^{\circ} \mathrm{C}$ for $6 \mathrm{~h}$ to allow shape recovery and selfhealing of the deactivated beams. The stiffnesses of the original and programmed structures (with deactivated beams) were measured using compression tests in the Instron mechanical tester $\left(\right.$ strain rate $=0.06 \mathrm{~s}^{-1}$ ).

\section{Vibration transformation of triangle lattices}

Triangle lattice structures were first fabricated using the abovementioned additive manufacturing process. The structures were placed on the top of a vibration generator (2185.00, Frederiksen) that was powered by a function generator (PI-8127, PASCO). Two accelerometers (352C22, PCB Piezotronics, USA) were attached on the bottom and top of the structures. Both accelerometers were connected to a signal conditioner $(482 \mathrm{C} 05, \mathrm{PCB}$ Piezotronics) to display the signal on an oscilloscope (TBS 1052B-EDU, Tektronix). The vibration transmittances of the lattice structures were measured as $\left|P_{\mathrm{t}} / P_{\mathrm{b}}\right|$, where $P_{\mathrm{t}}$ and $P_{\mathrm{b}}$ are the acceleration amplitudes of the top and bottom accelerometers, respectively. To transform a triangular lattice into a Kagome lattice, the horizontal beams were cut with a sharp blade and deactivated by bending at $80^{\circ} \mathrm{C}$ and cooling to room temperature. To transform the Kagome lattice back into a triangular lattice, the Kagome structure was heated to $80^{\circ} \mathrm{C}$ for $6 \mathrm{~h}$ to allow shape recovery and fracture healing.

\section{Acoustic transformation of lattice structures}

Lattice plates with small islands were designed and fabricated using the abovementioned additive manufacturing process. Three lattice plates were aligned and spaced $2 \mathrm{~cm}$ apart in a rectangular acrylate chamber (length of $30 \mathrm{~cm}$, height of $5 \mathrm{~cm}$ and width of $5 \mathrm{~cm}$, McMaster Carr). A loudspeaker (OT19NC00-04, Tymphany) connected to a function generator (PI-8127, PASCO) was placed at one end of the chamber to provide an acoustic signal. At the other end of the chamber, a microphone (378B02 with 426E01, PCB Piezotronics, USA) was used to collect the acoustic signal. The collected acoustic signal was processed by a signal conditioner (482C05, PCB Piezotronics) and displayed on a digital oscilloscope (TBS 1052B-EDU, Tektronix). The acoustic transmittance was measured as $\left|P_{\mathrm{w}} / P_{\mathrm{wo}}\right|$, where $P_{\mathrm{w}}$ and $P_{\text {wo }}$ were the measured acoustic pressures from the microphone with and without lattice structures, respectively. To program the lattice structures, one thin beam in each frame was cut with a sharp blade and deactivated by bending at $80^{\circ} \mathrm{C}$ and cooling to room temperature. To restore the structures, the programmed structures were heated to $80^{\circ} \mathrm{C}$ for $6 \mathrm{~h}$ to allow shape recovery and fracture healing.

\section{Acknowledgements}

Q.W. acknowledges the financial support from the Air Force Office of Scientific Research (FA9550-18-1-0192; Program manager: Dr. Ming-Jen Pan) and the National Science Foundation (CMMI-1762567). G.H. acknowledges the support from the Air Force Office of Scientific Research (AF 9550-15-1-0016; Program manager: Dr. Byung-Lip (Les) Lee). Y.C. is grateful for the financial support from the National Science Foundation (CMMI 1663663 and CMMI 1151191) and the Epstein Institute at the University of Southern California. 


\section{Author details}

${ }^{1}$ Sonny Astani Department of Civil and Environmental Engineering, University of Southern California, Los Angeles, CA 90089, USA. Epstein Department of Industrial and Systems Engineering, University of Southern California, Los Angeles, CA 90089, USA. ${ }^{3}$ Department of Mechanical and Aerospace Engineering, University of Missouri, Columbia, MO 65211, USA

\section{Author contributions}

K.Y. and Q.W. conceived the idea and designed the research. K.Y. and H.D. carried out experiments with the technical support of A.X., K.H.L., and Z.F. K.Y. and Q.W. carried out the numerical simulations and wrote the first manuscript. All authors contributed to interpreting the results and revising the manuscript

\section{Data availability}

All data needed to evaluate the conclusions in the paper are present in the paper and/or the Supplementary Materials. Additional data related to this paper may be requested from the authors.

\section{Conflict of interest}

The University of Southern California has filed a patent application related to the work described in this manuscript.

\section{Publisher's note}

Springer Nature remains neutral with regard to jurisdictional claims in published maps and institutional affiliations.

Supplementary information is available for this paper at https://doi.org/ 10.1038/s41427-020-0208-9.

Received: 13 December 2019 Accepted: 26 January 2020.

Published online: 27 March 2020

\section{References}

1. Gibson, L. J., Ashby, M. F. Cellular Solids: Structure and Properties. (CambridgeUniv. Press, 1999).

2. Wadley, H. N. Multifunctional periodic cellular metals. Philos. Trans. R. Soc. Lond A Math. Phys. Eng. Sci. 364, 31-68 (2006).

3. Fleck, N., Deshpande, V. \& Ashby, M. Micro-architectured materials: past, present and future. Proc. R. Soc. Lond. A Math. Phys. Eng. Sci. 466, 2495-2516 (2010).

4. Montemayor, L., Chernow, V. \& Greer, J. R. Materials by design: using architecture in material design to reach new property spaces. MRS Bull. $\mathbf{4 0}$ 1122-1129 (2015).

5. Schaedler, T. A. \& Carter, W. B. Architected cellular materials. Annu. Rev. Mater Res. 46, 187-210 (2016).

6. Jens, B. et al. Nanolattices: an emerging class of mechanical metamaterials Adv. Mater. 29, 1701850 (2017)

7. Roper, C. S. et al. Scalable 3D bicontinuous fluid networks: polymer heat exchangers toward artificial organs. Adv. Mater. 27, 2479-2484 (2015).

8. Xu, C., Gallant, B. M., Wunderlich, P. U., Lohmann, T. \& Greer, J. R. Threedimensional Au microlattices as positive electrodes for $\mathrm{Li}-\mathrm{O} 2$ batteries. ACS Nano 9, 5876-5883 (2015).

9. Surjadi, J. U. et al. Mechanical metamaterials and their engineering applications. Adv. Eng. Mater. 21, 1800864 (2019).

10. Bertoldi, K., Vitelli, V., Christensen, J. \& van Hecke, M. Flexible mechanical metamaterials. Nat. Rev. Mater. 2, 17066 (2017).

11. Bertoldi, K. Harnessing instabilities to design tunable architected cellular materials. Annu. Rev. Mater. Res. 47, 51-61 (2017).

12. Filipov, E. T., Tachi, T. \& Paulino, G. H. Origami tubes assembled into stiff, yet reconfigurable structures and metamaterials. Proc. Natl Acad. Sci. USA 112, 12321-12326 (2015)

13. Overvelde, J. T. et al. A three-dimensional actuated origami-inspired transformable metamaterial with multiple degrees of freedom. Nat. Commun. 7, 10929 (2016).

14. Overvelde, J. T., Weaver, J. C., Hoberman, C. \& Bertoldi, K. Rational design of reconfigurable prismatic architected materials. Nature 541, 347 (2017).
15. Kang, S. H. et al. Complex ordered patterns in mechanical instability induced geometrically frustrated triangular cellular structures. Phys. Rev. Lett. 112 098701 (2014).

16. Yu, K., Fang, N. X., Huang, G. \& Wang, Q. Magnetoactive acoustic metamaterials. Adv. Mater. 30, 1706348 (2018).

17. Fu, $\mathrm{H}$. et al. Morphable $3 \mathrm{D}$ mesostructures and microelectronic devices by multistable buckling mechanics. Nat. Mater. 17, 268 (2018).

18. Ge, Q. et al. Multimaterial $4 \mathrm{D}$ printing with tailorable shape memory polymers. Sci. Rep. 6, 31110 (2016)

19. Ding, Z. et al. Direct $4 \mathrm{D}$ printing via active composite materials. Sci. Adv. $\mathbf{3}$ e1602890 (2017).

20. Zarek, M. et al. 3D printing of shape memory polymers for flexible electronic devices. Adv. Mater. 28, 4449-4454 (2016).

21. Kotikian, A., Truby, R. L., Boley, J. W., White, T. J. \& Lewis, J. A. 3D printing of liquid crystal elastomeric actuators with spatially programed nematic order. Adv. Mater. 30, 1706164 (2018).

22. Zheng, $X$. et al. Ultralight, ultrastiff mechanical metamaterials. Science $\mathbf{3 4 4}$, 1373-1377 (2014)

23. Wang, Q. et al. Lightweight mechanical metamaterials with tunable negative thermal expansion. Phys. Rev. Lett. 117, 175901 (2016).

24. Wang, L., Lau, J., Thomas, E. L. \& Boyce, M. C. Co-continuous composite materials for stiffness, strength, and energy dissipation. Adv. Mater. 23 1524-1529 (2011).

25. Schaedler, T. A. et al. Ultralight metallic microlattices. Science 334, 962-965 (2011).

26. Eckel, Z. C. et al. Additive manufacturing of polymer-derived ceramics. Science 351, 58-62 (2016)

27. Meza, L. R., Das, S. \& Greer, J. R. Strong, lightweight, and recoverable threedimensional ceramic nanolattices. Science 345, 1322-1326 (2014).

28. Bauer, J., Schroer, A., Schwaiger, R. \& Kraft, O. Approaching theoretical strength in glassy carbon nanolattices. Nat. Mater. 15, 438-443 (2016).

29. Yu, K., Xin, A., Du, H., Li, Y. \& Wang, Q. Additive manufacturing of self-healing elastomers. NPG Asia Mater. 11, 7 (2019).

30. Kim, S. M. et al. Superior toughness and fast self-healing at room temperature engineered by transparent elastomers. Adv. Mater. 30, 1705145 (2018).

31. Chang, K., Jia, H. \& Gu, S.-Y. A transparent, highly stretchable, self-healing polyurethane based on disulfide bonds. Eur. Polym. J. 112, 822-831 (2019).

32. Binder W. H. Self-Healing Polymers: From Principles to Applications (John Wiley \& Sons, 2013).

33. Xu, B., Li, H., Wang, Y., Zhang, G. \& Zhang, Q. Nanocomposite hydrogels with high strength cross-linked by titania. RSC Adv. 3, 7233-7236 (2013).

34. Wu, D. Y., Meure, S. \& Solomon, D. Self-healing polymeric materials: a review of recent developments. Prog. Polym. Sci. 33, 479-522 (2008).

35. Blaiszik, B. et al. Self-healing polymers and composites. Annu Rev. Mater. Res. 40, 179-211 (2010)

36. Rae, P. \& Dattelbaum, D. The properties of poly (tetrafluoroethylene) (PTFE) in compression. Polymer 45, 7615-7625 (2004).

37. Kunwong, D., Sumanochitraporn, N. \& Kaewpirom, S. Curing behavior of a UVcurable coating based on urethane acrylate oligomer: the influence of reactive monomers. Sonklanakarin J. Sci. Technol. 33, 201 (2011).

38. Liu, C., Qin, H. \& Mather, P. Review of progress in shape-memory polymers. J. Mater. Chem. 17, 1543-1558 (2007)

39. Ling, L., Li, J., Zhang, G, Sun, R. \& Wong, C.P. Self-healing and shape memon linear polyurethane based on disulfide linkages with excellent mechanical property. Macromol. Res. 26, 365-373 (2018)

40. Xu, Y. \& Chen, D. A novel self-healing polyurethane based on disulfide bonds. Macromol. Chem. Phys. 217, 1191-1196 (2016).

41. Lei, Z. Q., Xiang, H. P., Yuan, Y. J., Rong, M. Z. \& Zhang, M. Q. RoomTemperature self-healable and remoldable cross-linked polymer based on the dynamic exchange of disulfide bonds. Chem. Mater. 26, 2038-2046 (2014).

42. Jian, X., Hu, Y., Zhou, W. \& Xiao, L. Self-healing polyurethane based on disulfide bond and hydrogen bond. Polym. Adv. Technol. 29, 463-469 (2018).

43. Yu, K., Xin, A. \& Wang, Q. Mechanics of self-healing polymer networks crosslinked by dynamic bonds. J. Mech. Phys. Solids 121, 409-431 (2018).

44. Wang, Q., Gao, Z. \& Yu, K. Interfacial self-healing of nanocomposite hydrogels: theory and experiment. J. Mech. Phys. Solids 109, 288-306 (2017).

45. Yu, K., Xin, A. \& Wang, Q. Mechanics of light-activated self-healing polymer networks. J. Mech. Phys. Solids 124, 643-662 (2019).

46. De Gennes, P.-G. Scaling Concepts in Polymer Physics (Cornell Univ. Press, 1979).

47. Rubinstein, M., Colby, R. Polymer Physics (Oxford Univ. Press, 2003). 
48. Deshpande, V. S., Fleck, N. A. \& Ashby, M. F. Effective properties of the octettruss lattice material. J. Mech. Phys. Solids 49, 1747-1769 (2001).

49. Phani, A. S., Woodhouse, J. \& Fleck, N. Wave propagation in two-dimensional periodic lattices. J. Acoust. Soc. Am. 119, 1995-2005 (2006).

50. Li, Y., Baker, E., Reissman, T., Sun, C. \& Liu, W. K. Design of mechanical metamaterials for simultaneous vibration isolation and energy harvesting. Appl. Phys. Lett. 111, 251903 (2017).

51. Ligon, S. C., Liska, R., Stampfl, Jr., Gurr, M. \& Mülhaupt, R. Polymers for 3D printing and customized additive manufacturing. Chem. Rev. 117, 10212-110290 (2017).
52. Sydney Gladman, A., Matsumoto, E. A., Nuzzo, R. G., Mahadevan, L. \& Lewis, J. A. Biomimetic 4D printing. Nat. Mater. 15, 413 (2016).

53. Tibbits, S. 4D printing: multi-material shape change. Architect. Des. 84, 116-121 (2014).

54. Kim, Y., Yuk, H., Zhao, R., Chester, S. A. \& Zhao, X. Printing ferromagnetic domains for untethered fast-transforming soft materials. Nature $\mathbf{5 5 8}, \mathbf{2 7 4 - 2 7 9}$ (2018).

55. Li, G. Q. \& Nettles, D. Thermomechanical characterization of a shape memory polymer based self-repairing syntactic foam. Polymer 51, 755-762 (2010). 